\title{
Mutual enrichment in ranked lists and the statistical assessment of position weight matrix motifs
}

\author{
Limor Leibovich ${ }^{1}$ and Zohar Yakhini ${ }^{1,2^{*}}$
}

\begin{abstract}
Background: Statistics in ranked lists is useful in analysing molecular biology measurement data, such as differential expression, resulting in ranked lists of genes, or ChIP-Seq, which yields ranked lists of genomic sequences. State of the art methods study fixed motifs in ranked lists of sequences. More flexible models such as position weight matrix (PWM) motifs are more challenging in this context, partially because it is not clear how to avoid the use of arbitrary thresholds.
\end{abstract}

Results: To assess the enrichment of a PWM motif in a ranked list we use a second ranking on the same set of elements induced by the PWM. Possible orders of one ranked list relative to another can be modelled as permutations. Due to sample space complexity, it is difficult to accurately characterize tail distributions in the group of permutations. In this paper we develop tight upper bounds on tail distributions of the size of the intersection of the top parts of two uniformly and independently drawn permutations. We further demonstrate advantages of this approach using our software implementation, $\mathrm{mmHG}$-Finder, which is publicly available, to study PWM motifs in several datasets. In addition to validating known motifs, we found GC-rich strings to be enriched amongst the promoter sequences of long non-coding RNAs that are specifically expressed in thyroid and prostate tissue samples and observed a statistical association with tissue specific CpG hypo-methylation.

Conclusions: We develop tight bounds that can be calculated in polynomial time. We demonstrate utility of mutual enrichment in motif search and assess performance for synthetic and biological datasets. We suggest that thyroid and prostate-specific long non-coding RNAs are regulated by transcription factors that bind GC-rich sequences, such as EGR1, SP1 and E2F3. We further suggest that this regulation is associated with DNA hypo-methylation.

Keywords: Statistical enrichment, Position weight matrices, High-throughput sequencing data analysis, Tissue specific methylation patterns, IncRNA

\section{Background}

Modern data analysis often faces the task of extracting characteristic features from sets of elements singled out according to some measurement. In molecular biology, for example, an experiment may lead to measurement results pertaining to genes and then questions are asked about the properties of genes for which these were high or low. This is an example, of course, and the set of

\footnotetext{
* Correspondence: zohar_yakhini@agilent.com

'Department of Computer Science, Technion - Israel Institute of Technology, Technion City, Haifa 32000, Israel

${ }^{2}$ Agilent Laboratories Israel, 94 Em Hamoshavot Road, 49527 Petach-Tikva, Israel
}

\section{Biomed Central}

elements does not have to be genes. They can be genomic regions, proteins, structures, etc. A central technique for addressing the analysis of characteristic properties of sets of elements is statistical enrichment. More specifically - the experiment results are often representable as ranked lists of elements and we then seek enrichment of other properties of these elements at the top or bottom of the ranked list. GSEA [1], for example, is a tool that addresses characteristic features of genes that are found to be differentially expressed in a comparative transcriptomics study. GOrilla [2,3] addresses GO terms enriched in ranked lists of genes where the ranking can be, for example, the result of processing 
differential expression data or of correlations computed between genomic DNA copy number and expression [4-6]. FATIGO [7] is also a tool that is useful in the context of analysing GO terms in ranked lists of genes. DRIMust [8-10] searches for sequence motifs that are enriched, in a statistically significant manner, in the top of a ranked list of sequences, which can be produced by techniques like ChIP-Seq.

All the aforementioned tools utilize a statistical approach that is based on assessing enrichment of an input set in an input ranked list by quantifying the enrichment obtained at various cutoffs applied to the ranked list. It is often the case, however, that two quantitative properties need to be compared to each other. For example, when the elements are genes, we may have measured differential expression values, as well as measured ChIPSeq signals. We are therefore interested in assessing mutual enrichment in two ranked lists. Another example consists of one ranking according to differential expression and one according to prediction scores for miRNA targets. miTEA $[11,12]$ addresses this latter case by statistically assessing the enrichment of miRNA targets in a ranked list of genes (also see [13]). To address mutual enrichment in two ranked lists over the same set of $N$ elements, miTEA [11] performs analysis on permutations. Mutual enrichment in the top of two ranked lists can be simplified, from a mathematical point of view, by arbitrarily setting the indices of one list to the identity permutation $(1,2, \ldots, N)$ and treating the other list as a permutation $\pi=\pi(1), \ldots, \pi(N)$ over these numbers. For the purpose of assessing the intersection of the top of the two ranked lists in a data driven manner, miTEA asks which prefix $\left[1, \ldots, n_{1}\right]$ is enriched in the first $n_{2}$ elements of $\pi$, that is in the set $\pi(1), \ldots, \pi\left(n_{2}\right)$. The statistics introduced by miTEA is called mmHG (minimum-minimum-Hyper-Geometric). A slightly different variant of $\mathrm{mmHG}$ is described later in this section.

Statistics in the group of permutations $S_{N}$ is often difficult because the number of entities to be considered by any null model is $N$ !. Direct exhaustive calculation of tail distributions over $S_{N}$ is therefore impractical for all but very small values of $N$. This difficulty is addressed by several heuristic techniques. Mapping into continuous spaces, such as in [14], has proven useful in certain cases but not for studying large deviations. In the case of enrichment statistics that focuses on the top of the permutation and seeks to assess extreme events, such as mmHG, we prefer to use bounds on tail probabilities. Tail probabilities are useful constructs when applied to analysing molecular biology measurement data as they enable statistical assessment of observed results.

In this work we derive tight bounds on the tail probabilities of mutual enrichment at the top of two random permutations uniformly drawn over $S_{N}$ and demonstrate the utility of this approach in the context of flexible motif discovery. Our bounds are computable in polynomial time and potentially add to the accuracy of reported position weight matrix (PWM) motifs for nucleic acid sequences.

\section{Mutual enrichment in ranked lists - the mmHG statistics}

The mmHG statistics [11] is a generalization of the $\mathrm{mHG}$ statistics [2,15-17]. The mHG statistics quantifies the enrichment level of a set of elements at the top of a ranked list of elements of the same type, whereas the mmHG statistics assesses the level of mutual enrichment in two ranked lists over the same set of elements. While any parametric or non-parametric correlation statistics (e.g. Spearman's correlation coefficient), that takes the same input, calculates the overall agreement between the two ranked lists, the mmHG statistic focuses only on agreement at the top of the two ranked lists. mmHG counts elements common to the top of both lists, without predefining what top is. Its intended output is the probability of observing an intersection at least as large in two randomly ranked lists (defined as the enrichment $p$-value). In this section we describe the mmHG statistics and in later sections we suggest tight bounds for the $p$-value. Our definition of the mmHG statistics varies slightly from that of Steinfeld et al. [11], which is used by miTEA.

Mutual enrichment in the top of two ranked lists can be simplified, from a mathematical point of view, by arbitrarily setting the indices of one list to the identity permutation $(1,2, \ldots, N)$ and treating the other list as a permutation. Details of this transform are given in the next section. We now define mmHG for the simple case of one permutation. Consider a permutation $\pi=$ $\pi(1), \ldots, \pi(N) \in S_{N}$ - the group of all permutations over the numbers $1, \ldots, N$. mmHG is a function that takes $\pi$ and calculates two numbers $1 \leq n_{1}, n_{2} \leq N$ such that the observed intersection between the numbers $1, \ldots, n_{1}$ and the first $n_{2}$ elements of $\pi$ - namely, $\pi(1), \ldots, \pi\left(n_{2}\right)$ - is the most surprising in terms of the hypergeometric $p$-value.

Formally, given $\pi \in S_{N}$ and for every $1 \leq n_{1}, n_{2} \leq N$, let $b_{\pi}\left(n_{1}, n_{2}\right)$ be the size of the intersection of $1, \ldots, n_{1}$ with $\pi(1), \ldots, \pi\left(n_{2}\right)$. Set

$$
\begin{aligned}
& m m H G \operatorname{score}(\pi) \\
& \quad=\min _{1 \leq n_{1} \leq N} \min _{1 \leq n_{2} \leq N} \operatorname{HGT}\left(N, n_{1}, n_{2}, b_{\pi}\left(n_{1}, n_{2}\right)\right)
\end{aligned}
$$

where HGT is the tail distribution of an hypergeometric random variable:

$$
\operatorname{HGT}\left(N, n_{1}, n_{2}, b\right)=\sum_{i=b}^{\min \left(n_{1}, n_{2}\right)} \frac{\left(\begin{array}{c}
n_{1} \\
i
\end{array}\right)\left(\begin{array}{c}
N-n_{1} \\
n_{2}-i
\end{array}\right)}{\left(\begin{array}{l}
N \\
n_{2}
\end{array}\right)}
$$


The mmHG score cannot be considered as a significance measure, due to the multiple testing involved in finding $n_{1}$ and $n_{2}$. A simple way to correct an mmHG score $s$ for multiple testing and report an upper bound on the $p$-value is to use the Bonferroni correction. Basically, $s$ is multiplied by the number of multiple tests conducted (which is $N^{2}$ ), yielding an upper bound on the p-value, as follows:

$$
\text { mmHG } p \text {-value }(s, N) \leq s \cdot N^{2}
$$

In the Results section we present significantly tighter bounds.

\section{Position weight matrix motifs}

Data produced by techniques such as ChIP-Seq [18], ChIP-exo [19], CLIP [20], PAR-CLIP [21] and others are readily representable as ranked lists of sequences, where the ranking is according to the measured binding affinity. Computational tools and approaches to motif discovery form part of the data analysis workflow that is used to extract knowledge and understanding from this type of studies. We are often interested in sequence motifs that are observed to be enriched in sequences where strong binding affinity is measured. A position weight matrix (PWM) is a commonly used representation of motifs in biological sequences [22-24]. This representation is more faithful to the underlying biology than representation by exact words, owing to the tendency of binding sites to be short and degenerate [25]. A PWM is a matrix of score values that gives a weighted match to any given substring of fixed length. It has one row for each symbol in the alphabet, and one column for each position in the pattern. Assuming an input sequence of length equal to the PWM width, we simply multiply the scores assigned to each letter in each of the positions in the input sequence to obtain the likelihood of the input string (alternatively, we can sum the logarithms of the probabilities). That is, the score assigned by a PWM to a substring $S=S_{1} \ldots S_{\mathrm{K}}$ is defined as $\prod_{j=1}^{K} p_{s_{j}, j}$, where $j$ represents a position in the substring; $s_{j}$ is the symbol at position $j$ in the substring; and $p_{\alpha, j}$ is the score in row $\alpha$, column $j$ of the matrix. In other words, a PWM score is the product of position-specific scores for each symbol in the substring. This definition can be generalized to yield a score for a sequence $S=S_{1} \ldots S_{\mathrm{M}}$ longer than the PWM by calculating $\max _{1 \leq i \leq M-K+1} \prod_{j=1}^{K} p_{s_{i+j-1}, j}$. Alternatively, an enhanced model that takes into account multiple occurrences of the PWM in the sequence can be applied by summing over sufficiently strong occurrences of the PWM or by other more sophisticated approaches [26].

\section{mmHG statistics for PWM motifs}

Given a set of sequences that were tested in a high throughput experiment such as ChIP-Seq [18], CLIP [20] and others, they can be ranked according to the measured binding affinities, yielding a ranked list $L_{1}$. Since usually we are interested in finding motifs amongst sequences having strong binding affinities, we actually search for motifs that are more prevalent at the top of this list. It is clear that any algorithm for de-novo flexible motif search would need to evaluate candidate PWMs. Given a PWM which we want to assess, the sequences can also be ranked according to their PWM scores, yielding another ranked list $L_{2}$, different from $L_{1}$. A significant PWM motif would yield significant scores for sequences having strong binding affinities. Therefore, the question of PWM motif discovery from ranked experimental data can be formulated as quantifying the mutual enrichment level for the two ranked lists $L_{1}$ and $L_{2}$. Given two ranked lists $L_{1}$ and $L_{2}$ over the universe of $N$ sequences, they can be transformed into two respective permutations, $\pi_{1}=\left(\pi_{1}(1), \ldots, \pi_{1}(N)\right)$ and $\pi_{2}=$ $\left(\pi_{2}(1), \ldots, \pi_{2}(N)\right)$. The relative permutation $\pi$, of $\pi_{2}$ w.r.t. $\pi_{1}$, is defined by $\pi\left(\pi_{1}(j)\right)=\pi_{2}(j)$, for every $j=1, \ldots, N$, or simply, using operations in the group $S_{N}: \pi=\pi_{2} \cdot \pi_{1}^{-1}$. Using the relative permutation $\pi$, we can represent the mutual enrichment of the top parts of $L_{1}$ and $L_{2}$ as $m m H G$ score $(\pi)$, defined above.

\section{Results}

Estimation of the mmHG $p$-value - introducing first upper bound - B1

Given an mmHG score $s$, observed in analysing real measurement data, we would like to assess the statistical significance of this observation. Assuming endless computational power, we would enumerate all permutations and calculate the mmHG score for each, in order to characterize the distribution of $\mathrm{mmHG}$ as a random variable over $S_{N}$. The $p$-value for $s$ is then simply:

$$
\begin{aligned}
& \text { mmHG } p \text {-value }(s, N) \\
& =\frac{\text { The number of permutations having mmHG score } \leq s}{N !}
\end{aligned}
$$

Since the number of permutations is huge, the process described above is very far from feasible. Therefore, we seek a computationally tractable upper bound, preferably tight.

A trivial upper bound is the Bonferroni corrected mmHG score defined by $s \cdot N^{2}$. A more subtle upper bound was suggested by Steinfeld et al. [11] and is briefly described later as bound B3. In this work we introduce tighter bounds that are polynomially computable.

We next describe an intuitive upper bound (B1) which we later refine to produce a tighter bound (B2). The input of the problem consists of an mmHG score $s$ and the total number of elements $N$. The output is an upper 
bound on the $p$-value. The efficiency of our approach relies on enumerating all possible HGT scores rather than enumerating all permutations in $S_{N}$. This approach is computationally efficient as HGT is a function of four input parameters: $N, n_{1}, n_{2}$, and $b$. Given $N$, there are $\mathrm{O}\left(N^{3}\right)$ possible combinations of $n_{1}, n_{2}$, and $b$. Also, given $N, n_{1}$ and $n_{2}, b$ can be any integer in the range $[\max (0$, $\left.\left.n_{2}-N+n_{1}\right), \min \left(n_{1}, n_{2}\right)\right]$. Next, all is left to do is to determine how many permutations correspond to each HGT score. To this end, let $\Lambda\left(N, n_{1}, n_{2}, b\right)$ be the number of permutations for which it holds that $b$ out of the first $n_{2}$ entries in the permutation are taken from the range $\left[1, \ldots, n_{1}\right]$. This formulation is equivalent to counting permutations for which we attain, at some point, the value $\operatorname{HGT}\left(N, n_{1}, n_{2}, b\right)$, had we taken the exhaustive approach. $\Lambda\left(N, n_{1}, n_{2}, b\right)$ can be represented as:

$$
\Lambda\left(N, n_{1}, n_{2}, b\right)=\left(\begin{array}{c}
n_{1} \\
b
\end{array}\right)\left(\begin{array}{c}
n_{2} \\
b
\end{array}\right) b !\left(\begin{array}{c}
N-n_{1} \\
n_{2}-b
\end{array}\right)\left(n_{2}-b\right) !\left(N-n_{2}\right) !
$$

as we first choose $b$ elements from the range $\left[1, \ldots, n_{1}\right]$ to appear at the first $n_{2}$ entries of the permutation (there are $\left(\begin{array}{c}n_{1} \\ b\end{array}\right)$ possibilities). Then, we choose the positions amongst the first $n_{2}$ entries that are occupied by these $b$ elements, while considering all internal arrangements (for each choice of $b$ elements there are $\left(\begin{array}{c}n_{2} \\ b\end{array}\right) b$ ! possibilities). We next choose $n_{2}-b$ elements from the range $\left[n_{1}+1, \ldots, N\right]$ to appear at the rest of the first $n_{2}$ entries of the permutation (there are $\left(\begin{array}{c}N-n_{1} \\ n_{2}-b\end{array}\right)$ possibilities for that) and consider all possible $\left(n_{2}-b\right)$ ! arrangements. Finally, we take into account all possible $\left(N-n_{2}\right)$ ! arrangements of the remaining $N-n_{2}$ entries of the permutation.

A straightforward upper bound for the number of permutations in $\mathrm{S}_{N}$ having mmHG score better than $s$ follows:

$$
\left|\left\{\pi^{\prime} \epsilon S_{N}: m m H G\left(\pi^{\prime}\right) \leq s\right\}\right| \leq \sum_{n_{1}, n_{2}, b: H G T\left(N, n_{1}, n_{2}, b\right) \leq s} \Lambda\left(N, n_{1}, n_{2}, b\right)
$$

From which an upper bound is easily derived:

$$
m m H G \text {-value }(s, N) \leq \frac{\sum_{n_{1}, n_{2}, b: H G T\left(N, n_{1}, n_{2}, b\right) \leq s} \Lambda\left(N, n_{1}, n_{2}, b\right)}{N !}
$$

By algebraic manipulations we get:

$$
m m H G \text {-value }(s, N) \leq \sum_{n_{1}, n_{2}, b: H G T\left(N, n_{1}, n_{2}, b\right) \leq s} \frac{\left(\begin{array}{c}
n_{1} \\
b
\end{array}\right)\left(\begin{array}{c}
N-n_{1} \\
n_{2}-b
\end{array}\right)}{\left(\begin{array}{c}
N \\
n_{2}
\end{array}\right)}
$$

This upper bound is simple and requires $\mathrm{O}\left(N^{3}\right)$ HGT calculations. An HGT calculation takes $\mathrm{O}(N)$ time, assuming binomial coefficients can be calculated in constant time. Constant time computation can be achieved using Stirling's approximation [27]: $\sqrt{2 \pi n}\left(\frac{\mathrm{n}}{\mathrm{e}}\right)^{\mathrm{n}} \mathrm{e}^{\frac{1}{12 \mathrm{n}+1}} \leq \mathrm{n} ! \leq$ $\sqrt{2 \pi n}\left(\frac{n}{e}\right)^{n} e^{\frac{1}{12 n}}$, which is tight for large factorials.

\section{A refined upper bound for the $p$-value - B2}

The upper bound introduced in the previous section counts the number of permutations for which the value $\operatorname{HGT}\left(N, n_{1}, n_{2}, b\right)$ is calculated when taking the nonpractical exhaustive approach that enumerates over all $N$ ! permutations. Ideally, we wish to count the number of permutations for which the value $\operatorname{HGT}\left(N, n_{1}, n_{2}, b\right)$ is also their mmHG score, as a permutation may correspond with many HGT values that are better than $s$, so it can be counted more than once. This explains why the formula introduced earlier is an upper bound and not an exact $p$-value. A second observation that follows is that the smaller the mmHG score $s$, the tighter the bound, because a permutation will have fewer combinations $(N$, $\left.n_{1}, n_{2}, b\right)$ having HGT values better than $s$.

Therefore, if we can reduce the extent of multiple counting of the same permutation, we will get a tighter bound. We do this by looking one step backwards. If, for example, $\operatorname{HGT}\left(N, n_{1}, n_{2}, b\right) \leq s$, we can exclude from the counting permutations that contain $b$ elements from the range $\left[1, \ldots, n_{1}-1\right]$ at their first $n_{2}$ entries because they are already taken into account in $\Lambda\left(N, n_{1}-1, n_{2}, b\right)$ (because necessarily $\operatorname{HGT}\left(N, n_{1}-1, n_{2}, b\right) \leq s$, as we will later explain).

Let $\psi\left(N, n_{1}, n_{2}, b\right)$ be the set of permutations for which it holds that $b$ out of the first $n_{2}$ entries are taken from the range $\left[1, \ldots, n_{1}\right]$ (note that $\Lambda\left(N, n_{1}, n_{2}, b\right)$ introduced earlier is, in fact, the size of $\left.\psi\left(N, n_{1}, n_{2}, b\right)\right)$. Assuming $\operatorname{HGT}\left(N, n_{1}, n_{2}, b\right) \leq s$, we can partition the set $\psi\left(N, n_{1}\right.$, $\left.n_{2}, b\right)$ into five disjoint subsets $\psi_{1}, \ldots, \psi_{5}$ such that $\psi=\psi_{1} \cup \psi_{2} \cup \psi_{3} \cup \psi_{4} \cup \psi_{5}$, as follows:

$$
\begin{aligned}
& \psi_{1}=\psi\left(N, n_{1}, n_{2}, b\right) \cap \psi\left(N, n_{1}-1, n_{2}-1, b-1\right) \cap \psi\left(N, n_{1}-1, n_{2}, b\right) \\
& \psi_{2}=\psi\left(N, n_{1}, n_{2}, b\right) \cap \psi\left(N, n_{1}-1, n_{2}-1, b-1\right) \cap \psi\left(N, n_{1}, n_{2}-1, b\right) \\
& \psi_{3}=\psi\left(N, n_{1}, n_{2}, b\right) \cap \psi\left(N, n_{1}-1, n_{2}-1, b-1\right) \cap \psi\left(N, n_{1}-1, n_{2}, b-1\right) \\
& \cap \psi\left(N, n_{1}, n_{2}-1, b-1\right) \\
& \psi_{4}=\psi\left(N, n_{1}, n_{2}, b\right) \cap \psi\left(N, n_{1}-1, n_{2}-1, b\right) \\
& \psi_{5}=\psi\left(N, n_{1}, n_{2}, b\right) \cap \psi\left(N, n_{1}-1, n_{2}-1, b-2\right) \\
& \cap \psi\left(N, n_{1}-1, n_{2}, b-1\right) \cap \psi\left(N, n_{1}, n_{2}-1, b-1\right)
\end{aligned}
$$

The properties of the hypergeometric distribution imply that given a tuple $\left(N, n_{1}, n_{2}, b\right)$, the permutations in $\psi_{1}, \psi_{2}, \psi_{4}$ can be disregarded from the current counting iteration. To explain why, we will demonstrate the argument on $\psi_{1}$. The permutations in $\psi_{1}$ contain $b$ elements from the range $\left[1, \ldots, n_{1}-1\right]$ at their first $n_{2}$ entries. Recall that we also assume that $\operatorname{HGT}\left(N, n_{1}, n_{2}, b\right) \leq s$. 
Therefore $\operatorname{HGT}\left(N, n_{1}-1, n_{2}, b\right) \leq s$ also holds, as the same intersection is observed for even a smaller set. Thus, the permutations in $\psi_{1}$ have already been counted as having HGT value better than $s$ when handling the triplet $n_{1}-1, n_{2}$ and $b$, and can be disregarded for the combination of $n_{1}, n_{2}$ and $b$. Similar arguments hold for $\psi_{2}$ and $\psi_{4}$.

The permutations in $\psi_{3}$ should be counted if three conditions hold: the first is $\operatorname{HGT}\left(N, n_{1}-1, n_{2}-1, b-1\right)$ $>s$; the second is $\operatorname{HGT}\left(N, n_{1}-1, n_{2}, b-1\right)>s$; and the third is $\operatorname{HGT}\left(N, n_{1}, n_{2}-1, b-1\right)>s$. Otherwise, the permutations in $\psi_{3}$ have been counted by former triplets. Similarly, the permutations in $\psi_{5}$ should be counted if the following three conditions hold: $\operatorname{HGT}\left(N, n_{1}-1, n_{2}-\right.$ $1, b-2)>s, \operatorname{HGT}\left(N, n_{1}-1, n_{2}, b-1\right)>s$, and $\operatorname{HGT}\left(N, n_{1}\right.$, $\left.n_{2}-1, b-1\right)>s$. Finally, we calculate the sizes of $\psi_{3}$ and $\psi_{5}$, in the relevant cases. The definition of $\psi_{3}$ implies that it consists of permutations that contain $b$-1 elements taken from the range $\left[1, \ldots, n_{1}-1\right]$ at their first $n_{2}-1$ entries, and also $n_{1}$ is positioned at entry $n_{2}$. Therefore:

$$
\left|\psi_{3}\right|=\left(\begin{array}{c}
n_{1}-1 \\
b-1
\end{array}\right)\left(\begin{array}{c}
n_{2}-1 \\
b-1
\end{array}\right)(b-1) !\left(\begin{array}{c}
N-n_{1} \\
n_{2}-b
\end{array}\right)\left(n_{2}-b\right) !\left(N-n_{2}\right) !
$$

Equivalently, the permutations in $\psi_{5}$ contain $b$-2 elements taken from the subset $\left[1, \ldots, n_{1}-1\right]$ at their first $n_{2}-1$ entries; $n_{1}$ is positioned at one of the first $n_{2}-1$ entries; and entry $n_{2}$ contains an element from $\left[1, \ldots, n_{1}-1\right]$. Therefore:

$$
\begin{aligned}
\left|\psi_{5}\right|= & \left(\begin{array}{c}
n_{1}-1 \\
b-2
\end{array}\right)\left(\begin{array}{c}
n_{2}-1 \\
b-2
\end{array}\right)(b-2) !\left(n_{2}-b+1\right)\left(\begin{array}{c}
N-n_{1} \\
n_{2}-b
\end{array}\right) \\
& \times\left(n_{2}-b\right) !\left(n_{1}-b+1\right)\left(N-n_{2}\right) !
\end{aligned}
$$

From the above we next conclude an upper bound. Denote

$$
\mathrm{I}\left(\operatorname{HGT}\left(N, n_{1}, n_{2}, b\right)>s\right)=\left\{\begin{array}{lr}
1, & \text { if } \operatorname{HGT}\left(N, n_{1}, n_{2}, b\right)>s \\
0, & \text { otherwise }
\end{array}\right.
$$

And let $\Lambda^{*}\left(N, n_{1}, n_{2}, b\right)=$

$$
\begin{aligned}
\left|\psi_{3}\right| & \times \mathrm{I}\left(\operatorname{HGT}\left(N, n_{1}-1, n_{2}-1, b-1\right)>s\right) \\
& \times \mathrm{I}\left(\operatorname{HGT}\left(N, n_{1}-1, n_{2}, b-1\right)>s\right) \\
& \times \mathrm{I}\left(\operatorname{HGT}\left(N, n_{1}, n_{2}-1, b-1\right)>s\right) \\
+ & \\
\left|\psi_{5}\right| & \times \mathrm{I}\left(\operatorname{HGT}\left(N, n_{1}-1, n_{2}-1, b-2\right)>s\right) \\
& \times \mathrm{I}\left(\operatorname{HGT}\left(N, n_{1}-1, n_{2}, b-1\right)>s\right) \\
& \times \mathrm{I}\left(\operatorname{HGT}\left(N, n_{1}, n_{2}-1, b-1\right)>s\right)
\end{aligned}
$$

We can thus derive the following upper bound for the $p$-value:

$$
\text { mmHG } p \text {-value }(s, N) \leq \frac{\sum_{n_{1}, n_{2}, b: H G T\left(N, n_{1}, n_{2}, b\right) \leq s} \Lambda^{*}\left(N, n_{1}, n_{2}, b\right)}{N !}
$$

Since $\Lambda^{*}$ is recursive, we need to define a base case. Recall that given $N, n_{1}$ and $n_{2}, b$ can be any integer in the range $\left[\max \left(0, n_{2}-N+n_{1}\right), \min \left(n_{1}, n_{2}\right)\right]$, hence determining a base case for $n_{1}$ and $n_{2}$ is sufficient $(N$ is known). The base case here is that when $n_{1} \leq 1$ or $n_{2} \leq 1$, $\Lambda^{*}\left(N, n_{1}, n_{2}, b\right)$ is defined the same as $\Lambda\left(N, n_{1}, n_{2}, b\right)$.

This upper bound uses more delicate counting than the bound B1 introduced in the previous section. In the following sections we assess the tightness of this bound. In later sections we demonstrate an application for PWM motif search.

\section{Comparison to a different mmHG variant - B3}

We note that the bound described in Steinfeld et al. [11] addresses a slightly different variant of $\mathrm{mmHG}$ as a random variable over $S_{N}$. The definition with which we work here is more amenable to deriving tight bounds as described above. Given a single permutation $\pi \in S_{N}$ and for every $i=1, \ldots, N$, a binary vector $\lambda_{i}$ is defined in which exactly $i$ entries are 1 and $N-i$ entries are 0 , as follows: $\lambda_{i}(j)=1$ iff $\pi(j) \leq i$. The mmHG score of a permutation $\pi$ is then defined by Steinfeld et al. [11] as:

$$
m m H G(\pi)=\min _{1 \leq i \leq \mathrm{N}} P-\operatorname{value}\left(m H G\left(\lambda_{i}\right)\right) \leq \min _{1 \leq i \leq \mathrm{N}} m H G\left(\lambda_{i}\right) \cdot i
$$

Where $\operatorname{mHG}\left(\lambda_{i}\right)=\min _{1 \leq n \leq N} H G T\left(N, i, n, b_{n}\right), \quad N=\left|\lambda_{i}\right|$ and $b_{n}=\sum_{k=1}^{n} \lambda_{i}(k)$. A possible upper bound is then given by:

\section{(*) $\quad P$-value $(m m H G(\pi)) \leq \min _{1 \leq i \leq \mathrm{N}} m H G\left(\lambda_{i}\right) \cdot i \cdot N$}

Computing the latter quantity requires $\mathrm{O}\left(N^{2}\right)$ HGT calculations, and is therefore computationally more efficient than the two bounds B1 and B2 of this current work (that require $\mathrm{O}\left(N^{3}\right)$ HGT calculations). We observed that our bound B2 was tighter than the bound in $(*)$, as later shown in Figure 1D. For example, for a permutation having mmHG score $=7.8 \cdot 10^{-25}(N=100)$, our bound was $3.5 \cdot 10^{-23}$ while $\left(^{*}\right)$ yielded $4.2 \cdot 10^{-21}$. For one permutation with $\mathrm{mmHG}$ score $=5.1 \cdot 10^{-5}(N=100)$, our bound was 0.026 while $\left(^{*}\right)$ yielded 0.2 . The latter example demonstrates that a tighter bound is important for classifying an observation as statistically significant (assuming a significance threshold of 0.05).

\section{Assessment of tightness}

In order to assess the quality of our bound B2, we compared it to the $p$-value, which can be calculated exactly for small values of $N$ (that is, in cases where $N$ ! is not too large) and empirically for larger values of $N$ (by randomly sampling permutations). Evidently, our bound B2 was significantly tighter than the Bonferroni bound for $N=10$ (Figure 1A) and $N=20$ (Figure 1B). We also observed that the smaller the mmHG scores - the tighter the bound, consistent with lesser over-counting 


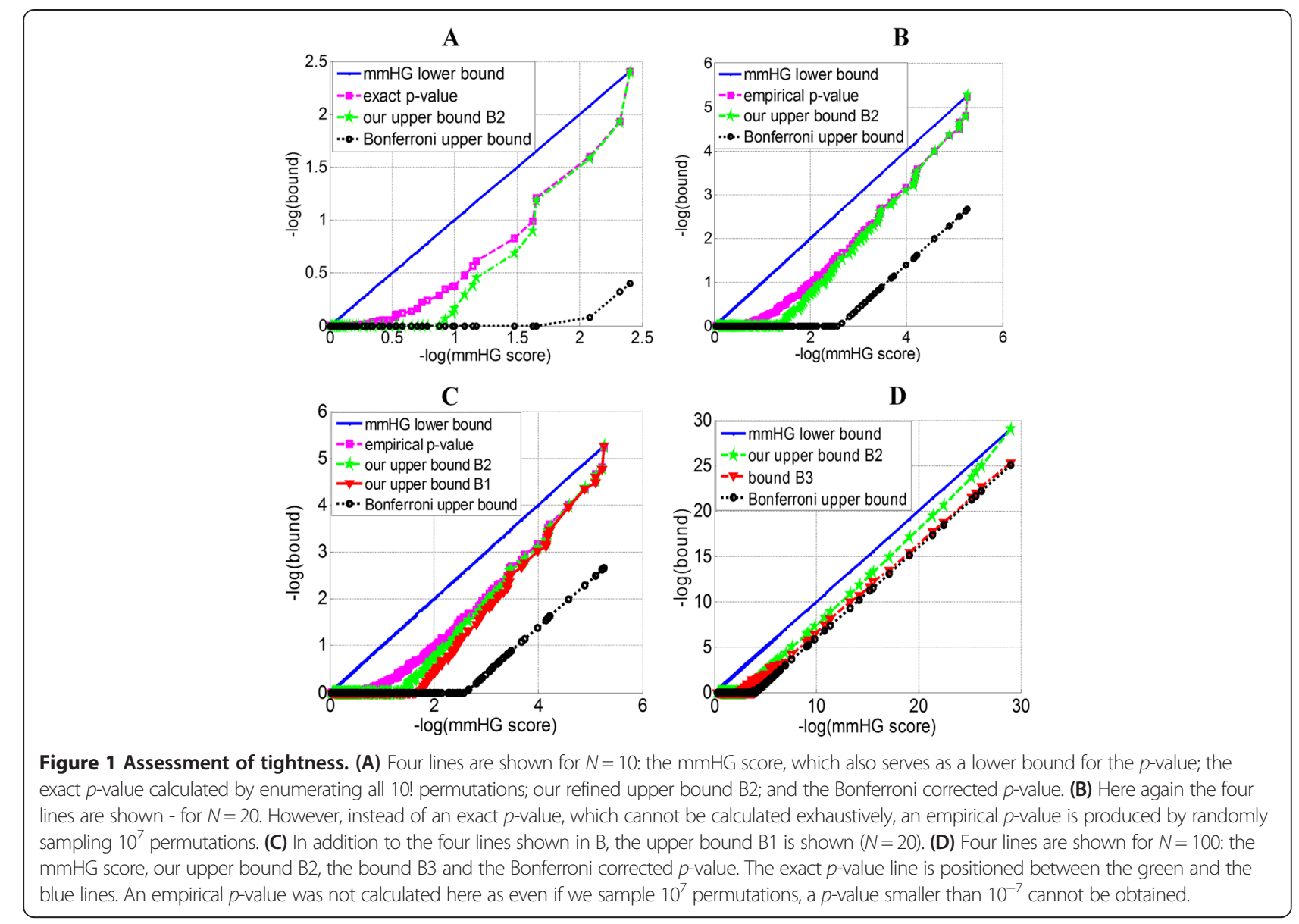

for smaller scores as explained in previous sections. Furthermore, our refined bound B2 is tighter than the bound B1 (Figure $1 \mathrm{C}$ ), and the latter is significantly better than the Bonferroni bound. Both bounds B1 and B2 are derived by enumerating HGT scores rather than enumerating permutations in $S_{N}$. The refinement of this approach produced by reducing the extent of multiple counting of permutation further improves the upper bound. In addition, the bound B2 was almost always observed to be tighter than the bound B3 (Figure 1D).

\section{An upper bound which balances between tightness and computational cost - B4}

The bound B2 is, evidently, very tight. It is, however, computationally heavy. We would still like to have an upper bound which is tighter than the Bonferroni bound and than the variant B3 but also faster to calculate. Such a compromise is achieved by generalizing an approach developed in [15] for the minimum hypergeometric statistics. Namely, given the number of elements $N$ and an attainable mmHG score $s$ for which we want to calculate the $p$-value, for each $1 \leq b \leq N$ and for each $1 \leq n_{1} \leq N$, let $n_{2}\left(b, n_{1}\right)$ be the maximal integer $n_{2}$ so that if in a permutation $\pi \in S_{N}, b$ out of the first $n_{2}$ entries in $\pi$ are taken from the range $\left[1, \ldots, n_{1}\right]$, then $\pi$ satisfies $\operatorname{HGT}_{\pi}\left(N, n_{1}, n_{2}, b\right) \leq s$. Monotonicity properties of the hyper-geometric distribution imply the existence of such $n_{2}$ integers. By definition, they are constants and independent of the original permutation for which the mmHG score $s$ was obtained. Due to monotonicity properties, given $b$ and $n_{1}$, the maximal value $n_{2}\left(b, n_{1}\right)$ can be calculated efficiently using binary search, which means that an upper bound that requires $\mathrm{O}\left(N^{2} \log N\right)$ calculations of HGT (instead of $\mathrm{O}\left(N^{3}\right)$ ) can be computed by using the following formula:

$$
m m H G \text {-value }(s, N) \leq \sum_{\mathrm{b}, n_{1}, n_{2}\left(\mathrm{~b}, n_{1}\right): H G T\left(N, n_{1}, n_{2}\left(\mathrm{~b}, n_{1}\right), b\right) \leq s} \frac{\left(\begin{array}{c}
n_{1} \\
b
\end{array}\right)\left(\begin{array}{c}
N-n_{1} \\
n_{2}\left(b, n_{1}\right)-b
\end{array}\right)}{\left(\begin{array}{c}
N \\
n_{2}\left(b, n_{1}\right)
\end{array}\right)}
$$


The performance of this bound, as well as of other bounds (in terms of tightness and running time), is demonstrated in Table 1. On average, this bound was 16.5 times tighter than the Bonferroni bound; B3 was approximately 7 times tighter than Bonferroni's bound, while B2 was 38 times tighter than Bonferroni's, on average. The average computation time for B4 was 3 minutes, in comparison with 1 second for B3 and 26 minutes for B2. We conclude that the bound $\mathrm{B} 4$ presented in this section may be a good compromise between tightness and computational cost compared with the other bounds introduced in this paper.

\section{Application in PWM motif search}

In this section we discuss mmHG as a framework for assessing the significance of PWM motifs in ranked lists. Given a ranked list of sequences and a PWM motif, by using the mmHG statistics and the bounds introduced earlier, we can

Table 1 Performance of various bounds

\begin{tabular}{|c|c|c|c|c|c|c|}
\hline Protein & $\mathrm{N}$ & mmHG score & Bound B2 & Bound B3 & Bound B4 & Bonferroni bound \\
\hline \multirow[t]{2}{*}{ TNWMNG } & 500 & $2.17 e-18$ & $2.75 e-14$ & $7.5 e-14$ & $6.28 \mathrm{e}-14$ & $5.43 e-13$ \\
\hline & & & $0.274 \mathrm{~min}$ & $0.0028 \mathrm{~min}$ & $0.079 \mathrm{~min}$ & \\
\hline \multirow[t]{2}{*}{ CTNNNAT } & 500 & $2.86 e-27$ & $1.32 \mathrm{e}-28$ & $3.66 e-28$ & $2.37 e-28$ & $2.86 e-27$ \\
\hline & & & $0.155 \mathrm{~min}$ & $0.0029 \min$ & $0.059 \mathrm{~min}$ & \\
\hline \multirow[t]{2}{*}{ MMMMMMMM } & 500 & $1.08 \mathrm{e}-43$ & $1.07 e-39$ & $3.47 e-39$ & $1.69 e-39$ & $2.71 e-38$ \\
\hline & & & $0.104 \mathrm{~min}$ & $0.003 \mathrm{~min}$ & $0.048 \mathrm{~min}$ & \\
\hline \multirow[t]{2}{*}{ REB1 } & 4000 & $1.66 \mathrm{e}-137$ & $9.18 e-133$ & $1.19 \mathrm{e}-131$ & $1.54 \mathrm{e}-132$ & $1.67 e-131$ \\
\hline & & & $17.25 \mathrm{~min}$ & $0.04 \min$ & $2.753 \mathrm{~min}$ & \\
\hline \multirow[t]{2}{*}{ CBF1 } & 4000 & $1.95 e-80$ & $9.15 e-76$ & $4.62 \mathrm{e}-75$ & $1.84 \mathrm{e}-75$ & $1.96 \mathrm{e}-74$ \\
\hline & & & $26.05 \mathrm{~min}$ & $0.03 \mathrm{~min}$ & $3.409 \min$ & \\
\hline \multirow[t]{2}{*}{ UME6 } & 4000 & $5.42 \mathrm{e}-88$ & $2.62 \mathrm{e}-83$ & $3.04 \mathrm{e}-82$ & $5.11 \mathrm{e}-83$ & $5.43 e-82$ \\
\hline & & & $23.81 \mathrm{~min}$ & $0.03 \mathrm{~min}$ & $3.374 \mathrm{~min}$ & \\
\hline \multirow[t]{2}{*}{ TYE7 } & 4000 & $1.62 \mathrm{e}-43$ & $5.63 e-39$ & $2.83 e-38$ & $1.39 e-38$ & $1.62 \mathrm{e}-37$ \\
\hline & & & $34.25 \mathrm{~min}$ & $0.02 \mathrm{~min}$ & $4.05 \mathrm{~min}$ & \\
\hline \multirow[t]{2}{*}{ GCN4 } & 4000 & $2.04 \mathrm{e}-50$ & $7.66 \mathrm{e}-46$ & $4.62 \mathrm{e}-45$ & $1.80 e-45$ & $2.04 \mathrm{e}-44$ \\
\hline & & & $35.43 \mathrm{~min}$ & $0.03 \mathrm{~min}$ & $3.95 \mathrm{~min}$ & \\
\hline \multirow[t]{2}{*}{ Puf5 } & 4795 & $7.91 \mathrm{e}-85$ & $3.38 \mathrm{e}-80$ & $5.60 \mathrm{e}-79$ & $6.95 e-80$ & 7.93e-79 \\
\hline & & & $31.51 \mathrm{~min}$ & $0.027 \mathrm{~min}$ & $4.51 \mathrm{~min}$ & \\
\hline \multirow[t]{2}{*}{ Pub1 } & 4251 & $1.49 e-84$ & $6.86 e-80$ & $1.33 e-78$ & $1.37 e-79$ & $1.5 e-78$ \\
\hline & & & $27.74 \mathrm{~min}$ & $0.033 \mathrm{~min}$ & $3.81 \mathrm{~min}$ & \\
\hline \multirow[t]{2}{*}{ Pab1 } & 4142 & $2.46 \mathrm{e}-11$ & $3.57 e-7$ & $5.17 e-7$ & $1.37 e-6$ & $2.46 e-5$ \\
\hline & & & $48.46 \mathrm{~min}$ & $0.007 \mathrm{~min}$ & $5.41 \mathrm{~min}$ & \\
\hline \multirow[t]{2}{*}{ Khd1 } & 4773 & $2.74 \mathrm{e}-20$ & $5.09 \mathrm{e}-16$ & $1.46 \mathrm{e}-14$ & $1.73 e-15$ & $2.74 \mathrm{e}-14$ \\
\hline & & & $47.58 \mathrm{~min}$ & $0.015 \mathrm{~min}$ & $5.84 \mathrm{~min}$ & \\
\hline \multirow[t]{2}{*}{ Nab2 } & 4101 & $2.09 \mathrm{e}-11$ & $3.08 \mathrm{e}-7$ & $1.48 \mathrm{e}-5$ & $1.18 \mathrm{e}-6$ & $2.09 e-5$ \\
\hline & & & $48.7 \min$ & $0.016 \mathrm{~min}$ & $5.34 \mathrm{~min}$ & \\
\hline \multirow[t]{2}{*}{ Vts1 } & 1787 & $1.44 \mathrm{e}-10$ & $4.74 \mathrm{e}-6$ & $1.33 e-5$ & $1.4 \mathrm{e}-5$ & $1.45 \mathrm{e}-4$ \\
\hline & & & $21.94 \mathrm{~min}$ & $0.003 \mathrm{~min}$ & $2.07 \mathrm{~min}$ & \\
\hline \multirow[t]{2}{*}{ Pin4 } & 4261 & $8.16 \mathrm{e}-14$ & $1.32 \mathrm{e}-9$ & $8.08 \mathrm{e}-9$ & $4.83 e-9$ & $8.18 \mathrm{e}-8$ \\
\hline & & & $49.38 \mathrm{~min}$ & $0.011 \mathrm{~min}$ & $5.48 \mathrm{~min}$ & \\
\hline \multirow[t]{2}{*}{ Nrd1 } & 3947 & $5.72 \mathrm{e}-12$ & $9.09 e-8$ & $5.71 \mathrm{e}-6$ & $3.36 \mathrm{e}-7$ & $5.74 \mathrm{e}-6$ \\
\hline & & & $47.67 \mathrm{~min}$ & $0.014 \mathrm{~min}$ & $5.11 \mathrm{~min}$ & \\
\hline \multirow[t]{2}{*}{ Yll032c } & 2286 & $1.06 e-9$ & $2.62 e-5$ & $1.61 \mathrm{e}-4$ & $8.3 e-5$ & 0.001 \\
\hline & & & $35.58 \mathrm{~min}$ & $0.003 \mathrm{~min}$ & $2.77 \mathrm{~min}$ & \\
\hline
\end{tabular}


assign a $p$-value to represent the significance of that PWM being enriched at the top of the list. To apply this approach for de-novo motif search, one needs to theoretically consider all possible PWMs. However, the search space - when considering position weight matrix motifs - is huge. Assuming the probabilities in the matrix are multiples of 0.1 and the alphabet is of size 4 , there are $286^{k}$ possible candidate PWMs of length $k$ (since each column must sum to 1 , the number of combinations in each column of the matrix is equal to the number of integer solutions for the equation $\mathrm{X}_{1}+\mathrm{X}_{2}+\mathrm{X}_{3}+\mathrm{X}_{4}=10$, which is $\left.\left(\begin{array}{l}13 \\ 10\end{array}\right)\right)$. Our approach to navigating in this search space is to narrow the search using the IUPAC alphabet, which considers all possible combinations of letters in the alphabet, and then represent the motif as a PWM based on its actual occurrences in the list. This heuristic approach, called mmHG-Finder, takes as input a ranked list of DNA or RNA sequences and returns significant motifs in PWM format. In cases where sequence ranking is not relevant or not available, it allows the use of positive and negative sets of sequences, searching for enriched motifs in the positive set using the negative set as the background.

We next describe the methodology implemented in mmHG-Finder. The input consists of a ranked list of sequences (or, alternatively, two sets of sequences representing target and background), as well as the motif width, given as a range $\left[k_{1}, k_{2}\right]$.

The algorithm:

1. Build a generalized suffix tree for the input sequences.

2. For each $k=k_{1}, \ldots, k_{2}$

- Traverse the tree to find all $k$-mers

- Sort the $k$-mers according to their enrichment at the top of the list (this is done using the mHG statistics), as explained in Leibovich et al. [8].

- Take the most significant fifty $k$-mers, to be used as starting points for the next step. This set of candidates is chosen such that the members are quite different. Note that this is a heuristic approach and the number 50 is somewhat arbitrary, chosen to succeed in catching the best performing PWMs without heavily paying in complexity.

- For each starting point, we iteratively replace one position in the $k$-mer by considering all possible IUPAC replacements and taking the one that improves the enrichment the most. We repeat this process for all positions several times, and eventually we get a motif in the IUPAC alphabet. We note that given an IUPAC pattern $P$, the occurrences of $P$ in the list are extracted efficiently by traversing the paths in the suffix tree that agree with $P$.
- Each IUPAC word is then expanded through a heuristic approach which is based on the Hamming neighbors of that word. Hamming neighbors are added as long as the new addition improves the enrichment $p$-value of the set of words, and as long as the overall similarity between the members in the set does not decrease below a similarity threshold. Since the neighbors are defined as exact words, they usually help in fine-tuning the correct weights of each letter in each position of the resulting PWM. Finally, the expanded motif is converted to a PWM.

3. The PWMs found in the previous step are assessed using the mmHG statistics and the best PWMs are returned as output, together with their $p$-value. The score assigned by a PWM to a string $S$ is the maximal score obtained for a substring of $S$. To obtain the likelihood of a substring of length $k$ (where $k$ is the PWM width), we simply multiply the scores assigned to each letter in each of the positions in that substring.

We provide an efficient implementation of the algorithm described above as publicly available software. Our application takes as input a ranked list of sequences and returns significant PWM motifs. It is compatible with all operating systems and can be freely downloaded from http://bioinfo. cs.technion.ac.il/people/zohar/mmHG-Finder-code/.

To evaluate the performance of mmHG-Finder in comparison to other state-of-the-art methods we ran it on 18 datasets -3 synthetically generated datasets and 15 generated from high throughput binding experiments (6 transcription factors and 9 RNA-binding proteins). Each synthetic dataset consisted of 500 randomly drawn sequences of length 100 . Then, variants of a predefined IUPAC motif were planted at the top 64 sequences of the dataset. We compared the motifs found by mmHGFinder to those obtained by using three other methods: the standard MEME program [28], DREME [29], and XXmotif [30]. Selected results of this comparison are summarized in Figure 2, and the full output is shown in Additional file 1. Evidently, mmHG-Finder outperformed all the other three tools on the synthetic examples, which contained degenerate motifs. DREME didn't find the motifs in any case, while MEME and XXmotif found a somewhat similar result in 1 out of the 3 tests. The other 15 examples were taken from DNA and RNA high-throughput experiments [31-33]. For 12 out of these 15 datasets, mmHG-Finder found the motifs which were compatible with the known literature motifs, and as the most significant result. In comparison, DREME found the known consensus in 11 cases; XXmotif detected the literature motif in 9 cases while MEME detected the known motif in 8 cases. In several datasets, such as for Pin4, mmHG-Finder identified the consensus 


\begin{tabular}{|c|c|c|c|c|}
\hline $\begin{array}{l}\text { The protein and } \\
\text { its consensus } \\
\text { binding motif }\end{array}$ & $\begin{array}{l}\text { mmHG- } \\
\text { Finder }\end{array}$ & MEME & DREME & XXmotif \\
\hline Synthetic & $6.28 \mathrm{e}-14$ & $2.2 \mathrm{e}+6$ & Nothing & $2.98 \mathrm{e}+00$ \\
\hline TNWMNG & T. 조 G & ending & found & 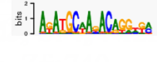 \\
\hline Synthetic & $2.37 \mathrm{e}-28$ & $5.8 \mathrm{e}+7$ & Nothing & $1.84 \mathrm{e}+01$ \\
\hline CTNNNAT & CT . AT & 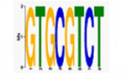 & found & 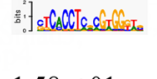 \\
\hline \multirow{2}{*}{$\begin{array}{l}\text { Synthetic } \\
\text { MMMMMMMM }\end{array}$} & $1.69 \mathrm{e}-39$ & \multirow{2}{*}{ 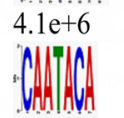 } & \multirow{2}{*}{$\begin{array}{l}\text { Nothing } \\
\text { found }\end{array}$} & \multirow{2}{*}{ 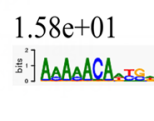 } \\
\hline & "AAAAACACE & & & \\
\hline P53 (DNA) & $\begin{array}{l}1.09 \mathrm{e}-174 \\
\mathrm{GA}_{\mathrm{A}} \mathrm{T} \mathrm{T} \text { 도우. }\end{array}$ & 4.6e-7 & $\begin{array}{l}4.9 \mathrm{e}-133 \\
\mathrm{ACATG} \mathrm{C}_{\mathrm{S}}\end{array}$ & 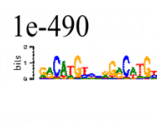 \\
\hline GCN4 (DNA) & $1.8 \mathrm{e}-45$ & $1.7 \mathrm{e}-16$ & $2.0 \mathrm{e}-32$ & $4.00 \mathrm{e}-17$ \\
\hline TGAsTCa & $\triangle T_{F} A_{\varepsilon} T C A$ & C & : TATATAT & 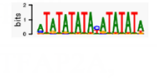 \\
\hline \multirow{3}{*}{$\begin{array}{l}\text { Puf5 (RNA) } \\
\text { UfUGUAAUAU }\end{array}$} & \multirow{3}{*}{$\begin{array}{l}6.95 \mathrm{e}-80 \\
\text { TGTA }\end{array}$} & \multirow{3}{*}{ 8.9e+1 } & $6.8 \mathrm{e}-42$ & $9.76 e-21$ \\
\hline & & & IIATATA & 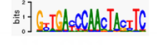 \\
\hline & & & $\begin{array}{l}3.1 \mathrm{e}-012 \\
\mathrm{~T} T \mathrm{~T}_{\mathrm{B}}\end{array}$ & $\begin{array}{l}1.61 \mathrm{e}-20 \\
\text { :IGTAA_A_IA }\end{array}$ \\
\hline $\begin{array}{l}\text { Pin4 (RNA) } \\
\cup \cup \cup A A \cup G A\end{array}$ & $\begin{array}{l}4.83 \mathrm{e}-9 \\
\text { TAெTG 수수. }\end{array}$ & 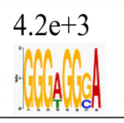 & $\begin{array}{l}3.1 \mathrm{e}-51 \\
\text { IATATA }\end{array}$ & 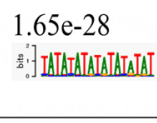 \\
\hline
\end{tabular}

Figure 2 Comparison between mmHG-Finder and other motif discovery tools. We evaluated the performance of mmHG-Finder in comparison to other state-of-the-art methods: MEME, DREME and XXmotif. Almost all input examples consisted of ranked lists, except for p53 (comprising target and background sets). Since MEME, DREME, and XXmotif expect to get a target set as input, we converted the ranked lists into target sets by taking the top 100 sequences for MEME (restricted by MEME's limitation of 60,000 characters) and the top 20\% sequences for the other tools. In the synthetic examples the entire ranked lists were taken as they are sufficiently small (to reflect useful comparison with MEME, as the motif is planted in top sequences, we had provided MEME, as input, with the ranking information by adding weights to the sequences, decreasing from 1 to 0 proportionally with the ranking). We used the default parameters in all comparison to other tools (e.g. zero-or-one-occurrence per sequence in MEME) and defined the expected motif length as the range 6 to 8 where possible (specifically, DREME and XXmotif do not have an input parameter for the motif length). Data and consensus motifs for p53 were taken from [31]; for REB1, CBF1, UME6, TYE7, GCN4 from [32]; and for the RNA binding proteins from [33]. Selected results are shown.

motif while other tools returned repetitive sequences as their top results. The mmHG statistics avoids such spurious results as they typically do not correlate with the measurement driven ranking.

\section{PWM motif search in long-non-coding RNA sequences}

We further analysed a collection of datasets comprising human long-non-coding (lnc) RNAs. LncRNA sequences were extracted and ranked according to the data reported by Cabili et al. [34]. Specifically, a stringent IncRNA set of 4662 loci was tested, where for each locus we know the expression levels in 19 different tissues. From these data we generated 19 lists, each ranked according to tissuespecificity. Given locus $i$ and tissue $j$, the tissue specificity score is defined as the difference between the expression of locus $i$ in tissue $j$ (denoted $\exp _{i, j}$ ) and the mean expression of locus $i$ (denoted as $\mu_{i}$ ). That said difference is measured in terms of the standard deviation of expression in locus $i$ (denoted as $\sigma_{i}$ ). Formally: tissue specificity score $_{i, j}=\frac{\exp _{i, j}-\mu_{i}}{\sigma_{i}}$

Calculating the above measure for all tissues reported in [34] yielded 19 ranked lists comprising 4360 lncRNAs (302 loci having standard deviation equal to zero were removed from the analysis). We then conducted three enrichment tests for each of these lists:

1. We searched for de-novo PWM motifs in the promoter sequences of the tissue-specific lncRNAs using $\mathrm{mmHG}$-Finder (introduced in the previous section). Promoter sequences were defined as 1000 bp upstream the transcription start site.

2. We scanned the promoter sequences of the tissue-specific lncRNAs with PWMs corresponding to known transcription factors, downloaded from the JASPAR database [35].

3. Independently of sequence, we calculated the statistical enrichment of measured transcription 
factor binding events within our lists of loci.

Transcription factor binding events within lncRNAs were downloaded from ChIP-Base database [36], which aggregates high-throughput sequencing data taken from hundreds of ChIP-Seq experiments.

Interestingly, almost all the motifs returned by mmHGFinder were GC-rich (Figure 3). In all three tests, the most significant results were obtained for thyroid-specific and prostate-specific lncRNAs. We further checked whether GC rich sequences are generally enriched amongst the promoter sequences of tissue specific lncRNAs by calculating the mutual enrichment between these two measures. The mutual enrichment between GC content and tissue specificity (Table 2) was the most significant for thyroid (mmHG $p$-value $\leq 3.9 \cdot 10^{-31}$ ), prostate $\left(5.8 \cdot 10^{-22}\right)$, adrenal $\left(5.5 \cdot 10^{-20}\right)$, brain $\left(1.6 \cdot 10^{-14}\right)$ and ovary $\left(8.8 \cdot 10^{-12}\right)$. Interestingly, Pearson's correlation between the GC content and the sequence rank was not observed to be strong (strongest correlation coefficient was -0.1), demonstrating that the overall agreement between two measures can be weak even when extremities agree.

\begin{tabular}{|c|c|c|}
\hline Tissue & mmHG-Finder output & $\begin{array}{l}\text { Transcription factors having } \\
\text { similar recognition sites }\end{array}$ \\
\hline Thyroid & $6.48 \mathrm{e}-37$ & $\begin{array}{l}\text { E2F3, E2F2, Zfp161, Zfx, SP1, } \\
\text { Egr1, Bc16b, K1f7, Sp4 }\end{array}$ \\
\hline Prostate & & $\begin{array}{l}\text { Bc16b, Egr1, Smad3, SP1, Nr2f2, } \\
\text { Zfp410, Mafb, Zfx, Zfp740 }\end{array}$ \\
\hline Brain & $1.54 \mathrm{e}-18$ & $\begin{array}{l}\text { Zfp161, E2F3, TFAP2A, E2F2, } \\
\text { Egr1, SP1, Myc, Sp4 }\end{array}$ \\
\hline Ovary & 2.11 & $\begin{array}{l}\text { Egr1, Nr2f2, Plag11, Bcl6b, Smad3, } \\
\text { SP1, Zfx, Zfp740 }\end{array}$ \\
\hline Foreskin & $7.37 \mathrm{e}$ & $\begin{array}{l}\text { Zfx, Nr2f2, Egr1, SP1, Zfp161, } \\
\text { TFAP2A, Smad3, Bcl6b, Sp100, } \\
\text { Zic1 }\end{array}$ \\
\hline Kidney & & $\begin{array}{l}\text { Egr1, SP1, Sp4, Klf7, Zfp281, } \\
\text { CTCF, INSM1, Zfp740 }\end{array}$ \\
\hline Breast & & $\begin{array}{l}\text { Egr1, Nr2f2, Zfx, TFAP2A, } \\
\text { Zfp161, Zic1, Plagl1, Zic2, Tcfap2a }\end{array}$ \\
\hline Adipose & & $\begin{array}{l}\text { Egr1, Tcfap2b, Plagl1, SP1, } \\
\text { NHLH1, INSM1, E2F2, Smad3, } \\
\text { Sp4 }\end{array}$ \\
\hline Adrenal & & $\begin{array}{l}\text { E2F3, E2F2, Myf6, Nr2f2, Plag11, } \\
\text { Sp4, Bcl6b, Smad3, CTCF, Zfp161 }\end{array}$ \\
\hline Lymph node & $2.01 \mathrm{e}-7$ & $\begin{array}{l}\text { Smad3, Sp4, Zfp161, E2F2, E2F3, } \\
\text { Glis2, INSM1, Egr1, SP1, Zfp740 }\end{array}$ \\
\hline Testes & $9.55 \mathrm{e}-6$ & $\begin{array}{l}\text { Zfp410, Fox11, Gm397, Six2, } \\
\text { Sox30 }\end{array}$ \\
\hline Liver & Nothing found & \\
\hline Lung & Nothing found & \\
\hline White blood cell & Nothing found & \\
\hline Colon & Nothing found & \\
\hline Heart & Nothing found & \\
\hline Skeletal muscle & Nothing found & \\
\hline Placenta & Nothing found & \\
\hline Lung fibroblasts & Nothing found & \\
\hline
\end{tabular}

Figure 3 Motifs in tissue-specific IncRNA promoter sequences. We analysed the promoter sequences of IncRNAs that are ranked according tissue-specificity. The motifs returned by mmHG-Finder are shown in the figure together with their $p$-value. We compared those motifs to known consensus motifs of transcription factors using TOMTOM [37] (motif database = JASPAR Vertebrates and UniPROBE Mouse) and the most significant results are shown (specifically, all similarity $p$-values are better than 0.018). 
Table 2 CpG hypo-methylation in tissue-specific IncRNA promoters

\begin{tabular}{|c|c|c|c|}
\hline \multirow[t]{2}{*}{ Tissue } & \multirow{2}{*}{$\begin{array}{l}\text { Mutual enrichment between } \\
\text { GC content and tissue-specificity }\end{array}$} & \multicolumn{2}{|c|}{ Mutual enrichment between hypo-methylation and tissue-specificity } \\
\hline & & Normal/subnormal cells & Cancer cells \\
\hline Thyroid & $3.89 e-31$ & No methylation data & No methylation data \\
\hline Prostate & $5.76 \mathrm{e}-22$ & 4.16e-11 (PrEC) & 0.002 (LNCaP) \\
\hline Adrenal & $5.46 e-20$ & No methylation data & No methylation data \\
\hline Brain & $1.57 \mathrm{e}-14$ & $1.21 \mathrm{e}-8(\mathrm{NH}-\mathrm{A})$ & $5.55 e-5(\cup 87)$ \\
\hline Ovary & $8.80 \mathrm{e}-12$ & No methylation data & 0.0085 (ovcar-3) \\
\hline Lymph node & $3.64 \mathrm{e}-6$ & No methylation data & No methylation data \\
\hline Adipose & $9.25 e-6$ & No methylation data & No methylation data \\
\hline Foreskin & $2.25 e-5$ & $0.72(\mathrm{BJ})$ & No methylation data \\
\hline \multirow[t]{2}{*}{ Breast } & $4.40 e-5$ & 5.08e-5 (HMEC) & $8.45 e-5$ (MCF7) \\
\hline & & 2.0e-10 (MCF10A) & 0.0065 (T-47D) \\
\hline Kidney & $6.34 \mathrm{e}-5$ & 1.56e-5 (HEK293) & No methylation data \\
\hline White blood cell & $3.78 \mathrm{e}-4$ & 0.6 (GM12878) & 0.21 (Jurkat) \\
\hline Placenta & 0.011 & No methylation data & No methylation data \\
\hline Colon & 0.012 & No methylation data & 1.0 (Сaco-2) \\
\hline Skeletal muscle & 0.04 & 0.34 (SKMC) & No methylation data \\
\hline Lung & 0.33 & No methylation data & No methylation data \\
\hline \multirow[t]{2}{*}{ Heart } & 1.0 & $1.0(\mathrm{HCM})$ & No methylation data \\
\hline & & 1.0 (HCF) & \\
\hline Liver & 1.0 & 1.0 (Hepatocytes) & 1.0 (HepG2) \\
\hline Testes & 1.0 & No methylation data & 1.0 (NT2-D1) \\
\hline \multirow[t]{2}{*}{ Lung fibroblasts } & 1.0 & 1.0 (IMR90) & No methylation data \\
\hline & & $1.0(\mathrm{AGO} 4450)$ & \\
\hline
\end{tabular}

We calculated the mutual enrichment between DNA hypo-methylation and tissue specificity for the IncRNA promoters. CpG methylation data was taken from UCSC Table Browser [39] (ENCODE/HAIB).

Furthermore, by intersecting the results of the second and the third tests together, we identified transcription factors that may regulate lncRNAs, mainly in thyroid and prostate. This set includes NRF1, E2F1, E2F3, E2F4, E2F6, EGR1, SP1, SP2 and ZBTB33. Moreover, the consensus recognition sites of EGR1, SP1 and E2F3 were found to be similar to the motifs returned by mmHG-Finder in thyroid, prostate and other tissues (Figure 3; the comparison was done using the motif discovery tool TOMTOM [37]). The full output of the second and the third tests are summarized in Additional file 2.

As GC-rich motifs may be associated with CpG methylation, and due to the possible binding of SP1 which has been suggested to protect $\mathrm{CPG}$ islands from de novo methylation $[17,38]$, we further tested the association between hypo-methylation and tissue specificity. For that, we downloaded genome wide (450 K) CpG Methylation data from UCSC Table Browser [39] (ENCODE/HAIB). We intersected lncRNA promoter regions with $\mathrm{CpG}$ methylation data, and continued only with the 1099 loci that were covered by the methylation experiment. For them, we calculated the mutual enrichment between hypo-methylation and tissue-specificity (the results are summarized in Table 2). Thyroid cells were not covered in this experiment, however two cell lines corresponding to prostate were tested (normal prostate epithelial cell line and cancerous prostate endodermal cell line). We observed that prostate-specific lncRNA promoters were less methylated than non-prostate-specific lncRNAs, and this was much stronger in normal cells than in cancer (mmHG $p$-value $\leq 4.16 \mathrm{e}-11$ in normal prostate cells, and 0.002 in prostate adenocarcinoma cells). We observed strong mutual enrichment between CpG hypo-methylation and tissue-specificity also in brain, ovary, breast and kidney. That is, significant mutual enrichment values were found for tissues where tissue-specific lncRNAs had GC-rich promoter sequences, but these values were not significant for tissues that did not show such GC-bias (heart, liver, testes, and lung fibroblasts). Additionally, in most cases the significance in normal cells was stronger than in cancer, which may be related to changes in methylation patterns acquired during carcinogenesis $[40,41]$. 


\section{Discussion}

The assessment of mutual enrichment in ranked lists is often required to support the analysis of biological measurement data, such as in the case of identifying sequence motifs that are involved in regulation processes. Relative ranking can be represented by using permutations over the measured elements. Therefore - the statistical assessment of mutual enrichment can be modelled by characterizing properties of random permutations. Due to the size of the measure space, statistics over $S_{N}$, the group of permutations over $N$ elements, is difficult to perform and implement. Mutual enrichment is more informative from the point of view of practical biological science than simple correlation measures, as it focuses on the top of the lists and not on the overall agreement, which may be weak even in cases where extremities agree. In this work we derive polynomially computable bounds for the associated tail distribution of mutual enrichment in ranked lists. Namely - we provide methods for computing an upper bound on the $p$-value of mutual enrichment at the top of two permutations uniformly and independently drawn over $S_{N}$. Naïve approaches to computing such bounds include variants of the Bonferroni approach. These do not provide tight bounds and may lead to mis-labeling results as non-significant. For several representative datasets, we note that our bound improves the Bonferroni derived $p$-value estimates by a factor of almost 40 , on average. Nevertheless, these improvements become relevant only for high $p$-values - for which significant scores should be treated with care anyway. We therefore note that the Bonferroni correction is applicable in many cases, as demonstrated in Table 1. Using our bounds is highly beneficial in borderline cases but is also important in cases where an accurate estimate of the $p$-value is desired, even if nuances do not affect the final biological research conclusions.

We use our statistical/algorithmic framework to support PWM motif searches and demonstrate the application to biological data. We identify motifs that characterize tissue specificity of lncRNA in thyroid and in prostate. Specifically, we find the EGR1 binding motif to be enriched in the promoter regions of IncRNAs which are thyroidspecific. EGR1 was observed to be highly expressed in thyroid (Additional file 1, taken from [36]), consistent with our stronger motif findings. Similarly, EGR1 is highly expressed in adipose tissue and its transcription factor binding sites are enriched in IncRNAs specific to this tissue. We do not have methylation data for the latter two tissues types. However - we do observe the promoters of lncRNAs that are specific to breast to have enriched occurrences of motifs that are similar to EGR1 transcription factor binding sites ( $p$-value of similarity according to TOMTOM $\left.=3 \cdot 52 \cdot 10^{-5}\right)$. EGR1 is also highly expressed in breast. Finally, the promoters of lncRNAs that are specific to breast are less methylated in breast (MCF10A and
HMEC cells) than other promoters. This suggests the role of EGR1 in driving tissue differentiation by transcribing tissue-specific lncRNAs and by protecting the associated promoters from methylation. EGR1 has been previously shown to recognize GC-rich consensus sequences located in CpG island promoters of active genes [42]. Generally, we observed that tissue-specific lncRNA promoters tend to be less methylated than those of non-tissue-specific lncRNAs in prostate, brain, ovary, breast and kidney, which may be associated with the GC-rich patterns enriched among their tissue-specific lncRNA promoter sequences.

Threshold-free alternatives to mmHG include the work of McLeay and Bailey, in which a linear regression method is applied [43]. It was shown to achieve high accuracy on a benchmark comprising 237 ChIP-chip datasets, which was higher than all other data driven methods tested, and specifically higher than Spearman's rank correlation. We note that applying linear regression or Spearman correlation to PWM motif search in ranked lists requires that for significant motifs we observe an overall agreement between the biological measurement and the PWM score. Nevertheless, the standard PWM formulation fails to predict binding affinity when the latter decreases to the point of non-specific binding [44]. In other words, the overall agreement between the PWM score and the binding affinity may be relatively weak. High correlation between the PWM score and the binding affinity needs to hold, in effect, only for sequences demonstrating high-binding affinity with respect to the protein of interest (that is, for sequences that are located at the top of the list) [45]. This weaker relationship is naturally addressed by the mmHG statistics. A combination of mmHG and a linear model, such as suggested in [43], applied to strong binders (top of the list), may yield an even more faithful and informative model.

Future research directions include more extensive application to biological data and the development of tighter and more efficient bounds. Our results show promise in enabling efficient and user-friendly PWM motif search in ranked lists. The software is freely avail able at http://bioinfo.cs.technion.ac.il/people/zohar/mmHGFinder-code/. Finally, the full characterization of the distribution of $\mathrm{mmHG}$ as a random variable over $\mathrm{S}_{N}$ remains an open question.

\section{Conclusions}

In this work we developed tight bounds on the tail distribution of mutual enrichment in ranked lists. Our bounds are computable in polynomial time and potentially add to the accuracy of reported results. We demonstrated the utility of mutual enrichment in motif search - specifically, when searching position weight matrix motifs in ranked lists, where the ranking can be according to binding affinity or according to any other biological measurement. 
Additionally, we used mutual enrichment to study tissuespecific long non-coding RNA regulation, and suggest that tissue-specific lncRNAs are regulated through GC-rich elements located on their promoters, in several tissue types. We hypothesize that these GC-rich patterns are associated with DNA hypo-methylation.

\section{Additional files}

Additional file 1: Table S1. Comparison between mmHG-Finder and other motif discovery tools. Figure S1. EGR1 expression profile.

Additional file 2: Table S2. The full output of the second and the third tests (including their intersection) for tissue-specific IncRNAs.

\section{Competing interests}

The authors declare that they have no competing interests.

\section{Authors' contributions}

$\mathrm{LL}$ derived the bounds, developed software and performed analysis. Both authors developed the PWM scoring approach, designed the study and wrote the manuscript. Both authors read and approved the final manuscript.

\section{Acknowledgements}

We thank Israel Steinfeld for critical and inspiring discussions. We thank Roy Navon for technical help with the software download service. We also thank the WABI anonymous reviewers for their useful comments. LL was partially supported by Israel Ministry of Science and Technology and by ISEF Fellowship.

Received: 1 December 2013 Accepted: 30 March 2014

Published: 5 April 2014

\section{References}

1. Subramanian A, Tamayo P, Mootha VK, Mukherjee S, Ebert BL, Gillette MA, Paulovich A, Pomeroy SL, Golub TR, Lander ES, Mesirov JP: Gene set enrichment analysis: a knowledge-based approach for interpreting genome-wide expression profiles. Proc Natl Acad Sci U S A 2005, 102:15545-15550.

2. Eden E, Navon R, Steinfeld I, Lipson D, Yakhini Z: GOrilla: a tool for discovery and visualization of enriched $\mathrm{GO}$ terms in ranked gene lists. BMC Bioinformatics 2009, 10:48.

3. GOrilla Webserver. [http://cbl-gorilla.cs.technion.ac.il/]

4. Ragle-Aure M, Steinfeld I, Baumbusch LO, Liestøl K, Lipson D, Nyberg S, Naume B, Sahlberg KK, Kristensen VN, Børresen-Dale A-L, Lingjærde OC, Yakhini Z: Identifying in-trans process associated genes in breast cancer by integrated analysis of copy number and expression data. PLOS ONE 2013, 8:e53014.

5. Akavia UD, Litvin O, Kim J, Sanchez-Garcia F, Kotliar D, Causton HC, Pochanard P, Mozes E, Garraway LA, Pe'er D: An integrated approach to uncover drivers of cancer. Cell 2010, 143:1005-1017.

6. Dehan E, Ben-Dor A, Liao W, Lipson D, Frimer H, Rienstein S, Simansky D, Krupsky M, Yaron P, Friedman E, Rechavi G, Perlman M, Aviram-Goldring A, Izraeli S, Bittner M, Yakhini Z, Kaminski N: Chromosomal aberrations and gene expression profiles in non-small cell lung cancer. Lung Cancer 2007, 56:175-184.

7. Al-Shahrour F, Díaz-Uriarte R, Dopazo J: FatiGO: a web tool for finding significant associations of gene ontology terms with groups of genes. Bioinformatics 2004, 20:578-580.

8. Leibovich L, Yakhini Z: Efficient motif search in ranked lists and applications to variable gap motifs. Nucleic Acids Res 2012, 40:5832-5847.

9. Leibovich L, Paz I, Yakhini Z, Mandel-Gutfreund Y: DRIMust: a web server for discovering rank imbalanced motifs using suffix trees. Nucleic Acids Res 2013, 41:W174-W179.

10. DRIMust Webserver. [http://drimust.technion.ac.il/]

11. Steinfeld I, Navon R, Ach R, Yakhini Z: miRNA target enrichment analysis reveals directly active miRNAs in health and disease. Nucleic Acids Res 2013, 41:e45-e45.
12. miTEA Webserver. [http://cbl-gorilla.cs.technion.ac.il/miTEA/]

13. Enerly E, Steinfeld I, Kleivi K, Leivonen S-K, Ragle-Aure M, Russnes HG, Rønneberg JA, Johnsen H, Navon R, Rødland E, Mäkelä R, Naume B, Perälä M, Kallioniemi O, Kristensen VN, Yakhini Z, Børresen-Dale A-L: miRNA-mRNA integrated analysis reveals roles for miRNAs in primary breast tumors. PLOS ONE 2011, 6:e16915.

14. Plis SM, Weisend MP, Damaraju E, Eichele T, Mayer A, Clark VP, Lane T, Calhoun VD: Effective connectivity analysis of fMRI and MEG data collected under identical paradigms. Comput Biol Med 2011, 41:1156-1165.

15. Eden E, Lipson D, Yogev S, Yakhini Z: Discovering motifs in ranked lists of DNA sequences. PLoS Comput Biol 2007, 3:e39.

16. Steinfeld I, Navon R, Ardigò D, Zavaroni I, Yakhini Z: Clinically driven semisupervised class discovery in gene expression data. Bioinformatics 2008, 24:i90-i97.

17. Straussman R, Nejman D, Roberts D, Steinfeld I, Blum B, Benvenisty N, Simon I, Yakhini Z, Cedar H: Developmental programming of CpG island methylation profiles in the human genome. Nat Struct Mol Biol 2009, 16:564-571.

18. Lee $B-K$, Bhinge $A A$, lyer VR: Wide-ranging functions of E2F4 in transcriptional activation and repression revealed by genome-wide analysis. Nucleic Acids Res 2011, 39:3558-3573.

19. Rhee Ho S, Pugh BF: Comprehensive genome-wide protein-DNA interactions detected at single-nucleotide resolution. Cell 2011, 147:1408-1419.

20. Lebedeva S, Jens M, Theil K, Schwanhäusser B, Selbach M, Landthaler M, Rajewsky N: Transcriptome-wide analysis of regulatory interactions of the RNA-binding protein HuR. Molecular Cell 2011, 43:340-352.

21. Hafner $M$, Landthaler $M$, Burger $L$, Khorshid $M$, Hausser J, Berninger $P$, Rothballer A, Ascano M Jr, Jungkamp A-C, Munschauer M, Ulrich A, Wardle GS, Dewell S, Zavolan M, Tuschl T: Transcriptome-wide identification of RNA-binding protein and MicroRNA target sites by PAR-CLIP. Cell 2010, 141:129-141.

22. Staden R: Computer methods to locate signals in nucleic acid sequences. Nucleic Acids Res 1984, 12:505-519.

23. Stormo GD, Schneider TD, Gold L: Quantitative analysis of the relationship between nucleotide sequence and functional activity. Nucleic Acids Res 1986, 14:6661-6679.

24. Hertz GZ, Stormo GD: Identifying DNA and protein patterns with statistically significant alignments of multiple sequences. Bioinformatics 1999, 15:563-577.

25. Tompa M, Li N, Bailey TL, Church GM, De Moor B, Eskin E, Favorov AV, Frith MC, Fu Y, Kent WJ, Makeev VJ, Mironov AA, Noble WS, Pavesi G, Pesole G, Regnier M, Simonis N, Sinha S, Thijs G, van Helden J, Vandenbogaert M, Weng Z, Workman C, Ye C, Zhu Z: Assessing computational tools for the discovery of transcription factor binding sites. Nat Biotech 2005, 23:137-144.

26. Sinha S: On counting position weight matrix matches in a sequence, with application to discriminative motif finding. Bioinformatics 2006 22:e454-e463

27. Abramowitz M, Stegun IA: Handbook of Mathematical Functions with Formulas, Graphs, and Mathematical Tables. New York: Dover Publications, Inc.; 1964.

28. Bailey TL, Elkan C: Unsupervised learning of multiple motifs in biopolymers using expectation maximization. Mach Learn 1995, 21:51-80.

29. Bailey TL: DREME: motif discovery in transcription factor ChIP-seq data. Bioinformatics 2011, 27:1653-1659.

30. Luehr S, Hartmann H, Söding J: The XXmotif web server for eXhaustive, weight matriX-based motif discovery in nucleotide sequences. Nucleic Acids Res 2012, 40:W104-W109.

31. Smeenk L, van Heeringen SJ, Koeppel M, van Driel MA, Bartels SJ, Akkers RC, Denissov S, Stunnenberg HG, Lohrum M: Characterization of genome-wide p53-binding sites upon stress response. Nucleic Acids Res 2008, 36:3639-3654.

32. Harbison CT, Gordon DB, Lee TI, Rinaldi NJ, Macisaac KD, Danford TW, Hannett NM, Tagne J-B, Reynolds DB, Yoo J, Jennings EG, Zeitlinger J, Pokholok DK, Kellis M, Rolfe PA, Takusagawa KT, Lander ES, Gifford DK, Fraenkel E, Young RA: Transcriptional regulatory code of a eukaryotic genome. Nature 2004, 431:99-104.

33. Hogan DJ, Riordan DP, Gerber AP, Herschlag D, Brown PO: Diverse RNA-binding proteins interact with functionally related sets of RNAs. Suggesting an extensive regulatory system. PLOS Biol 2008, 6:e255. 
34. Cabili MN, Trapnell C, Goff L, Koziol M, Tazon-Vega B, Regev A, Rinn JL: Integrative annotation of human large intergenic noncoding RNAs reveals global properties and specific subclasses. Genes Dev 2011, 25:1915-1927.

35. Mathelier A, Zhao X, Zhang AW, Parcy F, Worsley-Hunt R, Arenillas DJ, Buchman S, Chen C-y, Chou A, lenasescu H, Lim J, Shyr C, Tan G, Zhou M, Lenhard B, Sandelin A, Wasserman WW: JASPAR 2014: an extensively expanded and updated open-access database of transcription factor binding profiles. Nucleic Acids Res 2013, 42:D142-D147.

36. Yang J-H, Li J-H, Jiang S, Zhou H, Qu L-H: ChIPBase: a database for decoding the transcriptional regulation of long non-coding RNA and microRNA genes from ChIP-Seq data. Nucleic Acids Res 2013, 41:D177-D187.

37. Gupta S, Stamatoyannopoulos J, Bailey T, Noble W: Quantifying similarity between motifs. Genome Biol 2007, 8:R24.

38. Brandeis M, Frank D, Keshet I, Siegfried Z, Mendelsohn M, Names A, Temper V, Razin A, Cedar H: Sp1 elements protect a CpG island from de novo methylation. Nature 1994, 371:435-438.

39. UCSC Table Browser. [http://genome.ucsc.edu/cgi-bin/hgTables? command=start]

40. Bert SA, Robinson MD, Strbenac D, Statham AL, Song JZ, Hulf T, Sutherland RL, Coolen MW, Stirzaker C, Clark SJ: Regional activation of the cancer genome by long-range epigenetic remodeling. Cancer Cell 2013, 23:9-22

41. Nejman D, Straussman R, Steinfeld I, Ruvolo M, Roberts D, Yakhini Z, Cedar $\mathrm{H}$ : Molecular rules governing de novo methylation in cancer. Cancer Res 2014, 74:1475-1483.

42. Kubosaki A, Tomaru Y, Tagami M, Arner E, Miura H, Suzuki T, Suzuki M, Suzuki H, Hayashizaki Y: Genome-wide investigation of in vivo EGR-1 binding sites in monocytic differentiation. Genome Biol 2009, 10:R41.

43. McLeay R, Bailey T: Motif enrichment analysis: a unified framework and an evaluation on ChIP data. BMC Bioinformatics 2010, 11:165.

44. Frank DE, Saecker RM, Bond JP, Capp MW, Tsodikov OV, Melcher SE, Levandoski MM, Record MT: Thermodynamics of the interactions of lac repressor with variants of the symmetric lac operator: effects of converting a consensus site to a non-specific site. J Mol Biol 1997, 267:1186-1206.

45. Benos PV, Lapedes AS, Stormo GD: Is there a code for protein-DNA recognition? Probab(ilistical)ly. Bioessays 2002, 24:466-475.

doi:10.1186/1748-7188-9-11

Cite this article as: Leibovich and Yakhini: Mutual enrichment in ranked lists and the statistical assessment of position weight matrix motifs. Algorithms for Molecular Biology 2014 9:11.

\section{Submit your next manuscript to BioMed Central and take full advantage of:}

- Convenient online submission

- Thorough peer review

- No space constraints or color figure charges

- Immediate publication on acceptance

- Inclusion in PubMed, CAS, Scopus and Google Scholar

- Research which is freely available for redistribution 